Maria Effinger, Katja Leiskau und Annika-Valeska Walzel

\title{
All-In-One - arthistoricum.net auf dem Weg zum Fachinformationsdienst Kunst
}

\begin{abstract}
Zusammenfassung: Mit ,arthistoricum.net“ betreiben die UB Heidelberg und die SLUB Dresden seit Januar 2012 für ihre Sondersammelgebiete gemeinsam eine integrierte Virtuelle Fachbibliothek Kunst. Die Zusammenführung und Neukonzeption der Angebote stellte die strategischen Weichen für eine kooperative Weiterentwicklung. Ziele hierbei sind der Ausbau des konstruktiven Dialogs mit der Forschung und der Einsatz moderner Technologie im Sinne eines „Fachinformationsdienstes für die Wissenschaft“.
\end{abstract}

Schlüsselwörter: Kunstgeschichte; Sondersammelgebiete; Virtuelle Fachbibliothek

\section{All-In-One - arthistoricum.net and its transition to a Scientific Information Service for Art}

Abstract: In "arthistoricum.net” Heidelberg University Library and SLUB Dresden merged their special subject collections in 2012 to form one Virtual Library for Art. Both the union and the new concept have created a strategic foundation for further cooperative developments. The aims lie in the expansion of constructive dialogues with researchers and the employment of modern technology for the "Scientific Information Services".

Keywords: Art history, Special Subject Collections, Virtual Library

Dr. Maria Effinger: Effinger@ub.uni-heidelberg.de Dr. Katja Leiskau: Katja.Leiskau@slub-dresden.de Annika-Valeska Walzel M.A., M.A. (LIS): Annika-Valeska.Walzel@slub-dresden.de

\section{Erste Weichenstellung: Die virtuelle Fusion 2012}

Seit Januar 2012 sind die bislang getrennt präsentierten Angebote von „ViFaArt - Virtuelle Fachbibliothek für Gegenwartskunst“ und „arthistoricum.net - Virtuelle Fachbibliothek Kunstgeschichte“ in einer gemeinsamen Virtuellen Fachbibliothek Kunst unter dem Namen „arthistoricum.net" ${ }^{\text {"1 }}$ vereint. Damit ist nun erstmals eine umfassende medienübergreifende Recherche zum gesamten kunsthistorischen Themenspektrum mittels innovativer Suchtechnologie möglich.

Der Recherchezeitraum umfasst zum einen die frühchristliche und neuere Kunstgeschichte Europas und die von Europa beeinflusste Kunst in den USA, Kanada und Australien bis 1945 - verantwortet im Sondersammelgebiet „Kunstgeschichte bis 1945 und Allgemeine Kunstwissenschaft“ der Universitätsbibliothek Heidelberg² ${ }^{2}$. Zum anderen beinhaltet er die gesamte Geschichte der Kunst Europas und Nordamerikas ab 1945 als Bestandteile des Sondersammelgebiets „Zeitgenössische Kunst ab 1945, Fotografie, Industriedesign und Gebrauchsgrafik“ der Sächsischen Landesbibliothek - Staats- und Universitätsbibliothek Dresden ${ }^{3}$. Der Betrieb des Portals erfolgt in Kooperation mit dem Institut für Kunstgeschichte der Ludwig-Maximilians-Universität München (Lehrstuhl Prof. Dr. Hubertus Kohle) sowie weiteren Partnern.

\footnotetext{
$1 \mathrm{http} / /$ www.arthistoricum.net. Eine Übersicht der zur Virtuellen Fachbibliothek erschienenen Veröffentlichungen findet sich unter „Publikationen“.

2 Die UB Heidelberg betreut seit 1926 das Sondersammelgebiet „Kunstgeschichte bis 1945 und allgemeine Kunstwissenschaft“ (aktueller Bestand: rund 500.000 Monografien; ca. 1.500 laufende Zeitschriftenabonnements), vgl.http://SSG-Kunst.uni-hd.de. Die gemeinsam mit dem Zentralinstitut für Kunstgeschichte in München aufgebaute Virtuelle Fachbibliothek Kunstgeschichte „arthistoricum. net“ wurde von 2005 bis 2010 von der DFG gefördert.

3 Die SLUB Dresden hat als Zentralbibliothek für Kunst und Musik der DDR und SSG-Partner seit 1993 rund 195.000 Monografien zur Kunst seit 1945 einschließlich Fotografie, Industriedesign und Gebrauchsgrafik sowie annähernd 800 Zeitschriftenabonnements zusammengetragen. Die Virtuelle Fachbibliothek ViFaArt wurde von 2001 bis 2004 von der DFG gefördert.
} 


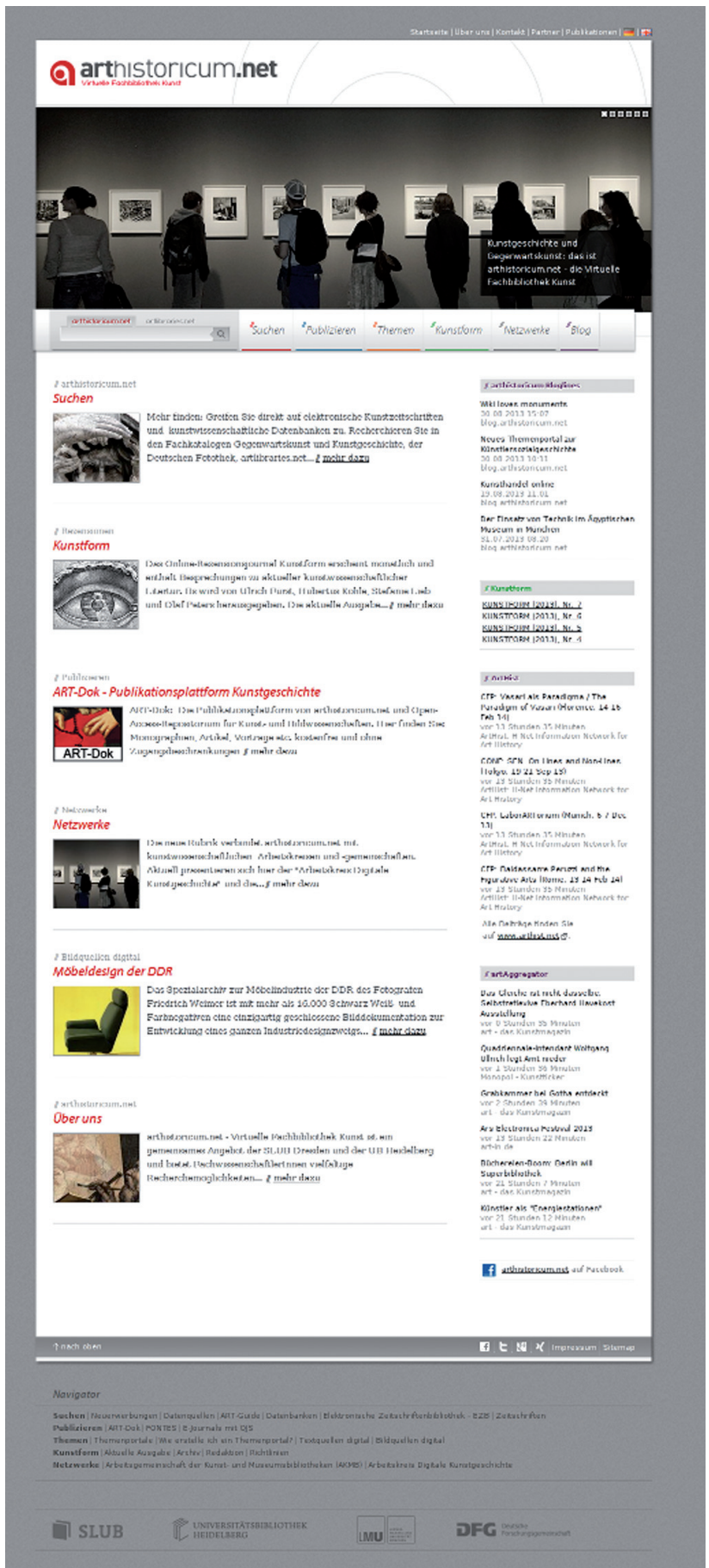

Abb. 1: Startseite von arthistoricum.net

Formal sicherlich gerechtfertigt, war die Parallelität der beiden Plattformen funktional jedoch problematisch. Für einen begrenzten Nutzerkreis in einem ohnehin dicht besetzten Informationsmarkt standen zwei eng wesensverwandte Informationsplattformen nebeneinander, weshalb bereits 2010, vor Abschluss der Evaluation der Sondersammelgebiete, die Entscheidung getroffen wurde, die beiden Virtuellen Fachbibliotheken zu einem integrierten Ange- bot zusammenzuführen und die Fusion in Eigenleistung $\mathrm{zu}$ realisieren.

Die Neukonzeption der gemeinsam gepflegten, weitgehend zweisprachig ausgelegten Plattform erfolgte unter Berücksichtigung der vormals in arthistoricum.net und ViFaArt vorhandenen Module. Angestrebt wurde eine grafisch und funktional überzeugende Summe aus den Angeboten beider Vorgängerportale. $\mathrm{Zu}$ diesem Zweck wurden diese einer kritischen Prüfung unterzogen, neu geordnet und zum Teil modifiziert. Das von der SLUB Dresden gehostete Content-Management-System TYPO-3 ermöglicht die kooperative Pflege der Portalinhalte und stellt die Basis des anlässlich des Relaunch modernisierten und klar strukturierten Portallayouts dar.

Die Praxis zeigte schnell, dass sowohl die konzeptionelle und strukturelle Weiterentwicklung als auch die redaktionelle Betreuung von arthistoricum.net an den beiden Standorten durch die gemeinsame virtuelle Arbeitsgrundlage reibungslos möglich ist, vom geringeren ökonomischen Aufwand einmal abgesehen. Die positive Resonanz der Nutzer sowie die Bereitschaft zahlreicher Wissenschaftler zur aktiven Beteiligung an den Portalinhalten belegen zudem den Erfolg dieses Konzepts. Im Kontext der aktuell geforderten Neuausrichtung der Sondersammelgebiete hin $\mathrm{zu}$ forschungsnahen, fachspezifischen Services $^{4}$ kann die strategische Zusammenführung der beiden Virtuellen Fachbibliotheken als zukunftsorientierte Weichenstellung verstanden werden.

Das in den folgenden Abschnitten beschriebene aktuelle Angebotsspektrum von arthistoricum.net reicht vor diesem Hintergrund von der Weiterführung bzw. dem Ausbau bereits etablierter Dienste bis zur Neukonzeption einzelner Module, wie beispielsweise der Rubrik „Netzwerke“.

\section{Integriertes Know-how}

Die Angebote der beiden Sondersammelgebiete und das zugehörige Know-how entwickelten sich bisher im Kontext der allgemeinen Informationsstruktur beider Häuser. Führt man die individuellen Entwicklungsstände und Produkte aus zwei Bibliotheken in einem gemeinsamen Dienst zusammen, bilden sich naturgemäß auch dort Zuständigkeitsschwerpunkte heraus: Es ergeben sich in einigen Bereichen Abgrenzungen, während man an anderen Stellen sehr eng zusammenarbeitet - und es entstehen beste Voraussetzungen für eine Win-win-Situation, die durch

4 Kümmel, Christoph: Nach den Sondersammelgebieten: Fachinformation als forschungsnaher Service. In: ZfBB 60 (2013), S. 5-15. 
Synergieeffekte auch Neuentwicklungen für das integrative Angebot realisierbar werden lässt. Die technische und konzeptionelle Weiterentwicklung wird auf mehrere Schultern verteilt und kommt dem gemeinsamen Informationsangebot ebenso zugute wie den betreibenden Bibliotheken. Diese Strategie erfordert allerdings neben einer effizienten dialogischen Kommunikation eine vorausschauende Planung und ist somit eher auf einen langfristigen Erfolg und nachhaltige Zusammenarbeit ausgerichtet als auf kurzfristigen Gewinn.

So konnte, um einen Eindruck von der Verteilung der Aufgaben und Nachnutzungsmöglichkeiten zu geben, bei der technischen Realisierung des Portals in der SLUB Dresden nicht nur auf den routinierten Betrieb des Content-Management-Systems TYPO-3 zurückgegriffen werden, sondern auch auf das in einem laufenden Projekt entwickelte Know-how zur maschinellen TiefenerschlieBung. ${ }^{5}$ Der Anspruch des Fachportals, möglichst viele relevante Daten unabhängig von ihrer Provenienz und Medienart in einer Suchoberfläche zu vereinen, konnte durch den Einsatz moderner Suchmaschinentechnologie $^{6}$ verwirklicht werden, die ebenfalls von der Dresdner Projektpartnerin bereitgestellt wurde. arthistoricum. net entwickelt sich damit zum nationalen Schaufenster mit medientypologisch übergreifenden Informationsangeboten - von Literaturnachweisen und Datenbanken bis hin zu Bildern und Volltexten. Zum Arbeitsprogramm seit dem Zusammenschluss zählte deshalb auch die Erweiterung des Suchraums um neue Datenquellen, wie die Einbindung von fachrelevanten Bibliothekskatalogen ${ }^{7}$ sowie die Fachausschnitte der Elektronischen Zeitschriftenbibliothek (EZB) und dem Datenbank-Infosystem DBIS. Einen weiteren Mehrwert gegenüber vergleichbaren Angeboten stellt die Integration von Volltexten der in Heidelberg digitalisierten kunsthistorischen Quellenliteratur sowie der umfangreichen Bildbestände der Deutschen Fotothek in Dresden dar. Während die SLUB die technische Weiterentwicklung der integrativen Suche betreibt, stellen Konzeption, Kommunikation mit Datenlieferanten sowie Daten- und Funktionstests wiederum eine gemeinsame Aufgabe beider Projektpartner dar. Als besondere Heidelberger Arbeitsschwerpunkte sind etwa

5 Vgl. Niederlein, Falk: Tiefenerschließung. Katalog in der 3. Dimension. In: BIS. Das Magazin der Bibliotheken in Sachsen, 6 (2013), Nr. 2, S. 92-95.

6 Zum Einsatz kommt das Discovery-System Primo der Firma Ex Libris.

7 Hierzu gehören zum Beispiel die Kataloge der Hamburger Kunsthalle, des documenta-Archivs Kassel oder der Kunstbibliothek der Staatlichen Museen zu Berlin. die - weiter unten ausführlicher beschriebenen - Komponenten „Elektronisches Publizieren“ sowie die Digitalisierung, Erschließung und Onlinebereitstellung kunsthistorischer Quellentexte im Open Access zu erwähnen. Im Laufe der letzten gut zehn Jahre konnte die UB Heidelberg mit ihrem hauseigenen, hochmodernen Digitalisierungszentrum sowie mit ihrer nationalen und internationalen Vernetzung Expertisen erwerben, die ein großes Innovationspotenzial bieten.

Neben diesen - quasi mit „in die Ehe“ gebrachten verteilten Kompetenzen stehen naturgemäß bei dem gemeinsamen Betrieb eines umfangreichen Portalangebots auch zahlreiche gemeinsame Aufgaben. Diese reichen von der Gesamtredaktion der Seiteninhalte über die Kommunikation mit den Kooperationspartnern bis hin zur Öffentlichkeitsarbeit. Und last but not least gehören dazu auch die gemeinsam verantwortete Entwicklungsstrategie, die kontinuierliche Evaluierung der Angebote ${ }^{8}$ oder die Bewältigung von Zukunftsaufgaben, wie beispielsweise Anforderungen der Langzeitarchivierung.

\section{Open Access - Publizieren und Digitalisieren}

Für die Verbreitung des Open Access-Gedankens in der deutschen Kunstgeschichte verfolgt arthistoricum.net vor allem zwei Wege: Zum einen fördert die Bereitstellung von komfortablen Publikationsplattformen und entsprechenden Workflows die Bereitschaft zum elektronischen Publizieren, und zum anderen wird durch die Digitalisierung überwiegend gemeinfreier, kunsthistorisch relevanter Literatur die Versorgungslage hinsichtlich des orts- und zeitunabhängigen Direktzugriffs auf Spezialliteratur sowie forschungsrelevante Informationen optimiert.

Bereits seit 2006 betreibt die UB Heidelberg den gut etablierten, kunstwissenschaftlichen Dokumentenserver ART-Dok ${ }^{9}$, der als Publikationsplattform in arthistoricum. net der internetbasierten Veröffentlichung, ErschlieBung und Archivierung kunstwissenschaftlicher Literatur dient. Bei den aktuell knapp 2.200 elektronisch im Open Access bereitgestellten Volltexten handelt es sich neben Erstpublikationen (z.B. in der Schriftenreihe „FONTES -

8 So wurde beispielsweise Anfang 2013 entschieden, den bislang im Rahmen der Virtuellen Fachbibliotheken gemeinsam gepflegten Fachinformationsführer „ART-Guide“ in dieser Form aufzugeben und zukünftig ausgewählte Internetquellen im zentralen Nachweisinstrument ,Zeitschriftendatenbank ZDB“ zu erschließen.

9 http://artdok.uni-hd.de. 
E-Quellen und Dokumente zur Kunst 1350-1750“10) auch um Zweitveröffentlichungen bereits gedruckt erschienener Schriften („Grüner Weg“). Bislang konnte für 29 namhafte Kunsthistorikerinnen und Kunsthistoriker ein großer Teil ihrer Publikationen elektronisch, in einer je individuellen Schriftenreihe gebündelt, bereitgestellt werden. Dafür werden von den Mitarbeitern der UB Heidelberg sowohl die Digitalisierung der Texte als auch die Erfassung und Erschließung in ART-Dok als Dienstleistung übernommen. Ein weiteres aktuelles über arthistoricum.net kommuniziertes Angebot ist die Unterstützung bei der Transformation bislang gedruckt erschienener Zeitschriften zu OpenAccess-E-Journals. Erste Erfahrungen konnten hier u.a. in enger Kooperation mit den Herausgebern und dem Verlag bei der Digitalisierung und Onlinestellung der etablierten Zeitschrift „kritische berichte - Zeitschrift für Kunst- und Kulturwissenschaften" "11 gewonnen werden.

In den Rubriken „Textquellen digital“ und „Bildquellen digital“ werden durch die Portalbetreiber historische Text- und Bildkollektionen frei verfügbar gemacht - die Auswahl wird in enger Verbindung mit der universitären Lehre und Forschung und in Kenntnis der Bedürfnisse der Leserschaft getroffen, um einen hohen Nutzungsgrad und gute Resonanz zu gewährleisten. Sowohl die SLUB Dresden als auch die UB Heidelberg verfügen über hauseigene, leistungsstarke Digitalisierungszentren.

Seit vielen Jahren digitalisiert die UB Heidelberg, da sie in ihrem Sammelschwerpunkt auf umfangreiche urheberrechtsfreie Bestände zurückgreifen kann, kunstwissenschaftliche Druckschriften. ${ }^{12}$ Alle Titel werden über arthistoricum.net unter der Rubrik „Textquellen digital“ oder im Kontext von Themenportalen bereitgestellt und dort systematisch um Kurzbiografien der Autoren, Werkeinführungen, Literaturhinweise sowie Internetlinks ergänzt. Die in digitalisierten Zeitschriften enthaltenen Aufsätze werden einzeln katalogisiert und auch über die zentrale Suche von arthistoricum.net recherchierbar gemacht. Aktuell sind rund 7.500 digitalisierte kunstwissenschaftliche Monografien sowie Zeitschriftenbände online verfügbar. ${ }^{13}$ In $\mathrm{Ab}$ grenzung zur reinen Massendigitalisierung liegt hier der strategische Schwerpunkt auf der Erstellung von qualitativ

$10 \mathrm{http}: / /$ www.arthistoricum.net/publizieren/fontes/.

11 http://kritische-berichte.uni-hd.de; Zum Einsatz kommt hier die Software Open Journal Systems (OJS) des Public Knowledge Projects (PKP).

12 Zur Ermöglichung eines reibungslosen und übersichtlichen Arbeitsprozesses setzt die Universitätsbibliothek Heidelberg das selbst entwickelte Workflow-Programm DWork für alle Teilschritte bei der Digitalisierung und der Webpräsentation ein, vgl. http://www.ub. uni-heidelberg.de/helios/digi/tech_workflow.html.

13 http://kunstliteratur-digital.uni-hd.de (ca. 1,25 Millionen Seiten). hochwertigen, intellektuell tiefenerschließenden Metadaten, welche die automatisierte Übernahme in nationale bzw. internationale Nachweisinstrumente und Portale ermöglichen. Die Titelauswahl erfolgt zum einen unter thematischen Gesichtspunkten „Kunst- und Satirezeitschriften des 19. und frühen 20. Jahrhunderts“ und „Auktionskataloge der Jahre 1901 bis 1945“ im Kontext von DFGgeförderten Projekten. Zum anderen wurden in den vergangenen Jahren in Eigenleistung kunstwissenschaftliche Monografien und Zeitschriftenbände (u.a. „Bücher zur Architektur und Gartenkunst“, „Quellen zur Geschichte der Kunstgeschichte") im Netz bereitgestellt; auch individuelle Nutzerwünsche werden berücksichtigt.

Auch in Dresden war neben dem klassischen Literaturerwerb die digitale Bereitstellung von Quellenmaterialien und forschungsrelevanten Daten für den dortigen Sammelschwerpunkt ein wichtiges Ziel der letzten Jahre ${ }^{14}$. Aufgrund des zeitlichen Rahmens dieses Sammelgebietes ab 1945 kann nicht oder nur in sehr geringem Maß auf urheberrechtsfreie Bestände zurückgegriffen werden. Ein starker Motor für die Erschließung und Bereitstellung von Werkabbildungen und Künstlerinformationen im Themenfeld Fotografie ist jedoch die Abteilung „Deutsche Fotothek", ein Universalbildarchiv zur Fotografie- und Kunstgeschichte mit mehr als 3 Millionen Fotografien. Seit September 2012 hat diese mit dem „Archiv der Fotografen. Schaufenster für deutsche Fotografie“"15 ihre Sammeltätigkeit intensiviert. Bereits erfolgreich akquiriert werden konnten fotografische Sammlungen von so prominenten Namen wie Wilfried Bauer, F.C. Gundlach, Heinz HajekHalke, Konrad Helbig, Peter Keetman, Ingrid von Kruse, Herbert List oder Michael Ruetz, deren Bestände online bisher überwiegend gar nicht oder nur über kommerzielle Bildagenturen recherchierbar waren.

Die kontinuierliche Integration von Ergebnissen vor allem drittmittelgeförderter Digitalisierungsprojekte in arthistoricum.net ist ein wichtiges strategisches Element, um die Attraktivität des Angebots für die Fachwissenschaft dauerhaft zu sichern. Auf diese Weise konnte in den letzten Jahren, trotz Ablauf der Förderung des Portals, dessen aktiver inhaltlicher Ausbau stetig vorangetrieben werden.

14 Die SLUB verwendet für ihren Digitalisierungsworkflow und zur Onlinepräsentation die Softwaresuite Goobi (Open Source) und entwickelt diese gemeinsam mit anderen Goobi-Anwendern weiter: http://www.slub-dresden.de/ueber-uns/projekte/infrastruktur-undsoftwareentwicklung/goobi-digital-library-modules/. 15 http://www.deutschefotothek.de/db/apsisa.dll/ete?action=view Page\&page=adf-info.xml. 


\section{4 Über den Tellerrand: Kooperationen und integrative Angebote}

Die Aktivitäten der UB Heidelberg und der SLUB Dresden bei der Ausgestaltung ihrer Sondersammelgebiete und dem Ausbau von arthistoricum.net erfolgen stets in engem Kontakt mit der Fachwissenschaft. Hohe Priorität bei der Auswahl von gedruckter und elektronischer Literatur oder aber retrodigitalisierter historischer Bestände haben dabei stets die Anforderungen der Fachcommunity. Als in dieser Hinsicht extrem wirkungsvolle Komponente für den lebendigen Dialog mit der Wissenschaft haben sich die vielen nationalen und internationalen Kooperationen erwiesen, die in der aktiven Mitarbeit zahlreicher Wissenschaftler bei arthistoricum.net im Kontext von Digitalisierungsprojekten oder im Bereich der Open Access-Publikationen mündeten.

Als Beispiele für eine besonders enge Kooperation zwischen Digitalisierung und Forschung können die Projekte der SLUB zur proletarischen Amateurfotografie der Weimarer Republik ${ }^{16}$ mit dem Institut für sächsische Landeskunde und Geschichte und zu den „Illustrierten Magazinen der klassischen Moderne“ (Projektpartner ist die Universität Erfurt) mit der Bereitstellung von 650 Ausgaben $^{17}$ genannt werden. Beide Projekte erschließen umfangreiche neue Quellen zur Fotografiegeschichte und Gebrauchsgrafik und bereichern damit das Sammelgebiet wie auch arthistoricum.net. In Heidelberg konnte in einem internationalen Kooperationsprojekt gemeinsam mit der Berliner Kunstbibliothek und dem Getty Research Institut das Themenportal „German Sales 1930-1945“18 aufgebaut werden: neben der Onlinestellung von gut 3.100 Auktionskatalogen ein umfassendes Informationsangebot zum Kunsthandel in der Zeit des Nationalsozialismus.

Neben der intensiven kooperativen Zusammenarbeit der beiden Betreiberinstitutionen mit diversen wissenschaftlichen Projektpartnern bei eigenen Drittmittelprojekten bietet arthistoricum.net auch externen Wissenschaftlern, Institutionen und Arbeitskreisen verschiedene frei gestaltbare Kooperationsmöglichkeiten an. Wichtige Elemente dieses kooperativen Angebots sind die sogenannten Themenportale, die einen vielschichtigen Zugang zu größeren gegeneinander abgrenzbaren Gegenständen oder

$16 \mathrm{http}: / /$ www.arbeiterfotografie-sachsen.de/.

$17 \mathrm{http} / / /$ www.illustrierte-presse.de.

$18 \mathrm{http} / /$ www.arthistoricum.net/themen/portale/german-sales/.
Zeiträumen der kunsthistorischen Forschung und Lehre vermitteln.

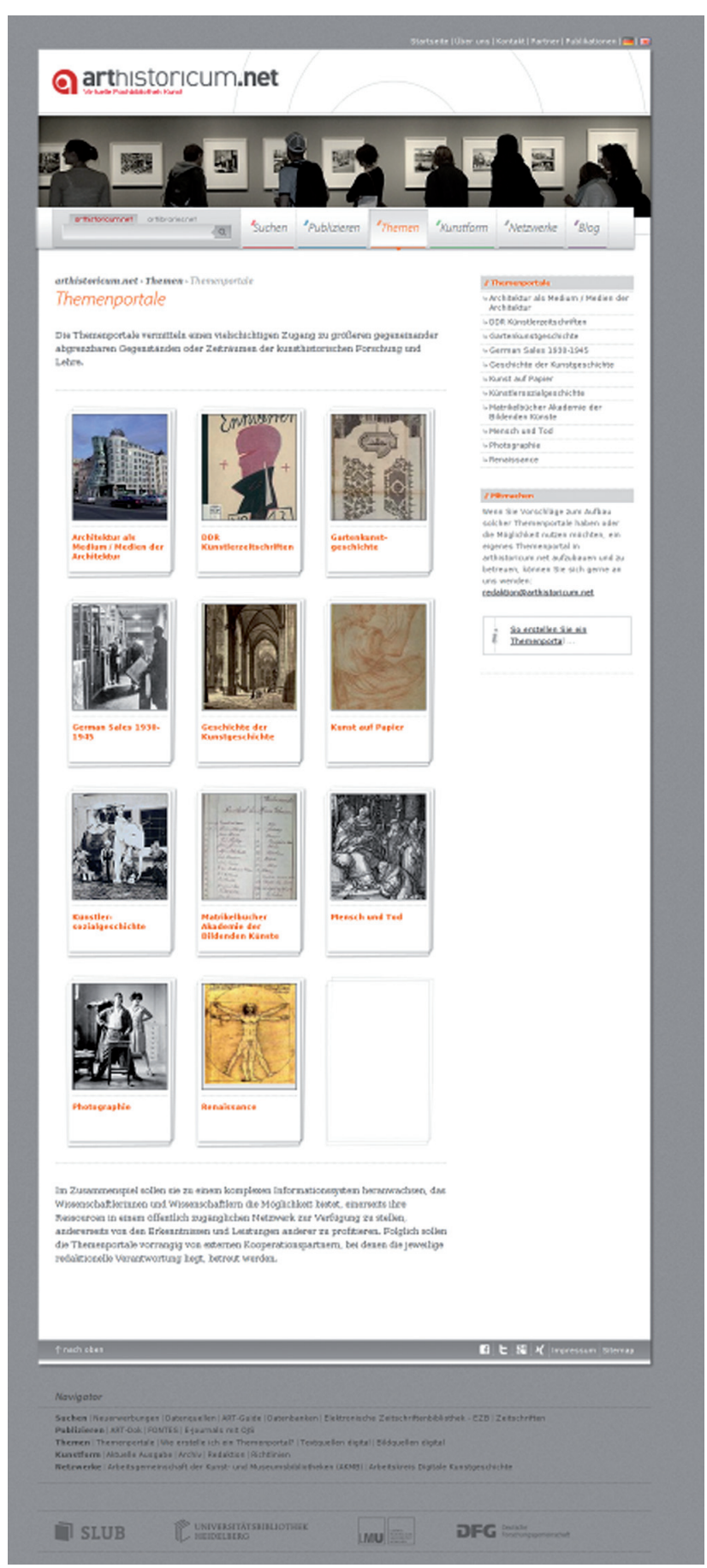

Abb. 2: Überblick über die Themenportale in arthistoricum.net

Die Themenportale sind genuine Module von arthistoricum.net - exklusive Angebote, die Projekte und Forschungsergebnisse oder auch Netzwerke in Bezug auf spezifische Fragestellungen darstellen können. Sie werden überwiegend von externen Kooperationspartnern geschaf- 
fen und gepflegt. Die redaktionelle Verantwortung kann allein bei Außenstehenden liegen oder auch gemeinsam mit den Sondersammelgebietsbibliotheken getragen werden, wie etwa bei „German Sales“. Insbesondere Nachwuchsforscher und außeruniversitäre Arbeitsgruppen erreichen mit diesen kostenfreien Angeboten eine bessere Sichtbarkeit und Vernetzung in einer nachhaltig verfügbaren und professionellen virtuellen Umgebung.

Zwei aktuelle Beispiele von bislang insgesamt 11 solcher Angebote seien hier genannt: Das Themenportal „Mensch und Tod“ wurde 2012 von der gleichnamigen Graphiksammlung der Heinrich-Heine-Universität Düsseldorf eingerichtet und versteht sich als Schnittstelle für Forschungen zu Grabmonumenten und Grablegen, zu Vanitas- und Memento-mori-Darstellungen und allen anderen Facetten dieses Themenkreises in unterschiedlichen Medien. ${ }^{19}$ In dem Anfang September 2013 freigeschalteten Themenportal „Künstlersozialgeschichte“20 gehen zwei Wissenschaftlerinnen der Universität Trier der Frage nach der Lebenswirklichkeit bildender Künstler von der Vormoderne bis heute nach.

Mit der Einführung des neuen Moduls „Netzwerke“ wurde das bewährte Modell der Themenportale inzwischen auch für Institutionen und Arbeitskreise geöffnet. Aktuell bieten sich hier Präsentationsräume für die Aktivitäten des Arbeitskreises „Digitale Kunstgeschichte“ und der „Arbeitsgemeinschaft der Kunst- und Museumsbibliotheken“ (AKMB). Letztere haben im März 2013 ihre Webseiten ganz in die Umgebung von arthistoricum.net integrieren lassen, um durch die Bündelung der Kompetenzen in dem gemeinsamen Kontakt- und Präsentationsraum arthistoricum.net Synergieeffekte in punkto Sichtbarkeit und direkter Informationsvermittlung zu erreichen.

Ganz neue Entwicklungsperspektiven und Impulse versprechen aktuelle Kooperationsvorhaben mit Informatikern der Heidelberger Universität zu den Themenbereichen „visuelle Objekterkennung“, „automatische Inhaltsanalyse von Bildern“ oder „Linked Open Data Infrastruktur zur Suche und Exploration kunstwissenschaftlicher Daten“.

\section{Keine Angst vor Blog, Facebook, Twitter und $\mathrm{Co}$.}

Blogs, Mikroblogs und soziale Netzwerke sind nicht nur im privaten Bereich vielgenutzte Kommunikationsmöglichkeiten, auch in Bereichen der institutionellen Öffentlichkeitsarbeit oder der wissenschaftlichen Fachkommunikationen spielen sie eine immer größere Rolle. Bereits seit 2009 bot arthistoricum.net einen Blog als Wordpress-Instanz an, der nach der Fusion der beiden Virtuellen Fachbibliotheken ebenfalls in das CMS TYPO-3 überführt wurde. Dies ermöglichte eine Anpassung an das Portal-Layout und machte den Blog zu einem unverzichtbaren, interaktiven Teil der fachspezifischen Informationsinfrastruktur.

Wie schon in Bezug auf die Themenportale ausgeführt, bietet auch der Betrieb eines Blogs eine ideale Möglichkeit der Einbindung der kunsthistorischen Wissenschaftler in das Portal: Als Autoren für die einzelnen Kategorien konnten jeweils Spezialisten für die verschiedenen Fachrichtungen gewonnen werden. Insgesamt setzt sich das Team aktuell aus rund 30 wissenschaftlichen Autoren zusammen. Alle Beiträger werden im Umgang mit dem System geschult und erhalten die Möglichkeit, auf einer individuellen Unterseite die eigenen Kontaktdaten sowie bei Bedarf weiterführende Informationen $\mathrm{zu}$ platzieren. Im Blog von arthistoricum.net wird laufend über aktuelle kunstwissenschaftliche Themen berichtet und diskutiert - in der Regel auf hochqualifiziertem Niveau. Das Spektrum ist weit gefasst: von der mittelalterlichen zur digitalen Kunst, von der Denkmalpflege bis zur kunsthistorischen Ausbildung und Fragen der digitalen Kunstgeschichte oder auch zu Problemen der Universitäts- und Bildungspolitik.

Neben diesem eigenen Informations- und Kommunikationskanal werden die dort diskutierten Inhalte, Themen und Neuigkeiten des Fachportals auch über Facebook und Twitter verbreitet. ${ }^{21}$ Steht bei dem Blog der direkte wissenschaftliche Austausch von Meinungen, Fragen und Kommentaren im Fokus, sind Mikroblog und soziales Netzwerk hervorragende Möglichkeiten der Öffentlichkeitsarbeit, um eine erhöhte Sichtbarkeit und Aufmerksamkeit für Fachportale und Fachthemen zu erzeugen und vor allem die studentischen NutzerInnen abzuholen.
19 https://www.arthistoricum.net/themen/portale/mensch-undtod/.

20 https://www.arthistoricum.net/themen/portale/kuenstlersozial geschichte/.
$21 \mathrm{https} / /$ www.facebook.com/arthistoricum.net (gestartet Anfang 2012, bis 30.08.2013 insgesamt 543 „Gefällt mir“-Angaben); Tweets von @arthistoricum gibt es seit Beginn 2013. 


\section{Nur wer sich ändert, bleibt sich treu}

\section{1 arthistoricum.net auf dem Weg zum Fachinformationsdienst für die Wissenschaft}

Die Intention des neuen DFG-Förderprogramms „Fachinformationsdienste für die Wissenschaft (FID)“ bietet für die beiden Partnerinstitutionen UB Heidelberg und SLUB Dresden ideale Voraussetzungen, das kooperative Angebot durch den stetigen Ausbau des konstruktiven Dialogs mit der Wissenschaft und durch den Einsatz moderner Technologie in eine weitere Dimension zu führen.

Nach all dem bereits gemeinsam Erreichten lag es auf der Hand, den Ende Mai bei der DFG einzureichenden Antrag zum Aufbau eines „Fachinformationsdienst Kunst“ nicht wie bislang für jedes bisherige Sondersammelgebiet getrennt $\mathrm{zu}$ stellen, sondern auch hier die Kräfte zu bündeln und einen gemeinsamen Antrag vorzulegen und damit das in der Fachcommunity bereits gut etablierte „arthistoricum.net“ als gemeinsame Marke für dieses Angebot weiter auszubauen. Um die bereits vorhandenen Angebote noch expliziter an den Bedürfnissen der Fachcommunity ausrichten und arthistoricum.net zu einem „Fachinformationsdienst Kunst“ im Sinne der aktuellen Fördervorgaben konsequent weiterentwickeln und ausbauen zu können, wurden im Projektantrag für die Jahre 2014 bis 2016 sechs Aktionsfelder definiert.

Die vorbehaltlich einer positiven Begutachtung des Antrags auszubauenden bzw. neu zu entwickelnden Mehrwertdienste in den Bereichen „Suchtechnologie“, „Ausbau der Informationsinfrastruktur“, „Elektronisches Publizieren“; „Retrodigitalisierung und Onlinepräsentation forschungsrelevanter Medienbestände“ sowie „Wissenschaftskommunikation" gehen sowohl in der Qualität wie auch in der Quantität weit über die lokale Informationsinfrastruktur anderer wissenschaftlicher Bibliotheken mit kunstwissenschaftlichem Angebot hinaus. Dabei sollen die in den letzten Jahren aufgebauten Kooperationen mit Wissenschaftlern in zahlreichen Teilprojekten erweitert und intensiviert werden. Handlungsmaxime ist dabei vor allem bei der Bereitstellung technischer Tools und Funktionalitäten sowie dem Angebot fachspezifischer Informationsdienstleistungen -, vorausschauend die Bedürfnisse der Fachinteressenten aufzugreifen und zu erfüllen. Der bereits rege genutzte Blog soll auch weiterhin den spartenübergreifenden fachlichen Austausch fördern und interdisziplinären Forschungs- und Interessengruppen in enger Anbindung an arthistoricum.net einen Präsentationsraum bieten.

Basierend auf dem bereits bestehenden Netzwerk soll der „Fachinformationsdienst Kunst“ von einem wissenschaftlichen Beirat begleitet werden. Dieser wird - zusammen mit den bibliothekarischen Vertretern - die Leitlinien für den Betrieb und die Weiterentwicklung des FID festlegen und so eine kontinuierliche Qualitätssicherung gewährleisten. Der Beirat soll Anfang 2014 seine Arbeit aufnehmen, einmal jährlich mit den Projektverantwortlichen in Dresden und Heidelberg zusammenkommen sowie fortlaufend die Entwicklungen mittels Mail- und Blogkommunikation begleiten.

\subsection{Neue Aspekte beim Ausbau der Fachinformationsinfrastruktur}

Ein innovatives Ziel der Weiterentwicklung und Pflege von arthistoricum.net ist es, der Fachcommunity über ein gemeinsames Recherchewerkzeug einen direkten Zugriff auf fachwissenschaftlich relevante Publikationen aller Medienformen bis auf die Volltextebene anzubieten. Dies führt über die bisherigen Möglichkeiten der bibliografischen Literaturrecherche deutlich hinaus. Dabei sollen neue Recherchefunktionalitäten weiteren fachwissenschaftlichen Content (sowohl aus frei verfügbaren Digitalisaten als auch aus lizenzierten Datenquellen beider Trägereinrichtungen) durchsuchbar machen.

Damit spezifische Nutzergruppen auch von außerhalb des Campusnetzes auf den digitalen Content aus lizenzierten Datenbanken und Textkollektionen zugreifen können, ist es notwendig, einen Authentifizierungsmechanismus in das Fachportal zu integrieren. Durch die Anbindung an die Autorisierungs- und Authentifizierungsinfrastruktur des Deutschen Forschungsnetzes DFN-AAI mittels Shibboleth kann der Durchgriff auf lizenzierte Volltexte ortsunabhängig und barrierefrei erfolgen.

Als Ergänzung zu den bereits bestehenden Angeboten von arthistoricum.net ${ }^{22}$ sollen schrittweise u.a. folgende weitere Quellen integriert werden: Getty Research Portal, Nationallizenzen (z.B. Art Sales Catalogues, Art Bibliography Modern, International Bibliography of Art) sowie weitere kunsthistorische Datenbanken. Die bereits abgeschlossene erfolgreiche Integration der in der EZB nachgewiesenen kunstwissenschaftlichen Zeitschriften und Datenbanken aus DBIS in die Suche von arthistoricum.net

22 http://www.arthistoricum.net/suchen/datenquellen/. 
soll dabei im Zuge des Authentifizierungsworkflows um eine Verfügbarkeitsanzeige auf Titelebene ergänzt werden.

Ein weiteres Element zur Steigerung des Such- und Nutzungskomforts von arthistoricum.net besteht in der Optimierung seiner Anzeige auf mobilen Endgeräten. Mit der allgemeinen Zunahme mobiler Endgeräte (Tablets, Smartphones etc.) in der Wissenschaft ist im Bereich der Literaturrecherche bereits eine signifikant erhöhte mobile Nutzung zu verzeichnen. Um das gesamte Portalangebot (Suche, Blog, Themen u.a.) effizient mobil verfügbar zu machen, sollen auf der Basis von HTML5 und Responsive Design Zugriffe mit mobilen Endgeräten ohne spezielle Software (Apps) möglich sein.

Um zukünftig die FID-Kunden automatisch, regelmäßig und individuell über neu in arthistoricum.net eingebrachte Erwerbungen, fachrelevante Datenbanken, EJournals, E-Books, Retrodigitalisate u.v.m. informieren zu können, ist die Einrichtung eines „Profildienst FID Kunst“ vorgesehen. Nutzer von arthistoricum.net können sich nach erfolgter Registrierung individuell ihr gewünschtes Portfolio zusammenstellen und festlegen, in welchen Abständen sie per Mail oder RSS-Feed über Neuzugänge informiert werden wollen. Zusätzlich soll eine Personalisierung der Suchfunktion ergänzt werden, um so eigene Recherchen besser zu überblicken.

Ein weiterer Baustein im konsequenten Ausbau der Informationsinfrastruktur des Fachs kann perspektivisch die Anbindung eines Forschungsverzeichnisses Kunst an arthistoricum.net darstellen, das als (Personen-)Verzeichnis zum Gesamtbereich der Kunstwissenschaften, institutionenübergreifend Kontaktdaten, Expertisen und Forschungsschwerpunkte vereint. Zwar besteht über den Verband Deutscher Kunsthistoriker (VdK) ein Branchenbuch zu (kommerziellen) Dienstleistungen der Kunstwissenschaft ${ }^{23}$, dieses schließt jedoch Forschung und Lehre nicht ein. Mit dem neuen Modul des Fachinformationsdienstes ließen sich Mehrwerte für die Fachcommunity durch eine vereinfachte Vernetzung und zentralen Zugriff auf aktuelle Forschungs-Kontaktdaten schaffen, die semiautomatisch aus bestehenden Informationen von Institutswebseiten aggregiert werden können. Durch Verknüpfung mit der GND, semantische Anreicherung der Daten und Bereitstellung als Linked Open Data erhöhen sich Anbindung und Sichtbarkeit der fachwissenschaftlichen Gemeinschaft.

23 http://www.kunsthistoriker.org/service.html.

\subsection{Optimierung der Erwerbungsstrategien}

Die neue Förderpolitik der DFG führt im Bereich des Bestandsaufbaus zu einem „Paradigmenwechsel“ und stellt damit die Verantwortlichen vor neue Herausforderungen: An die Stelle des Vollständigkeitsprinzips im Sinne einer Reservoir-Funktion tritt nun die Orientierung an nachgewiesenen Bedarfen und aktuellen Forschungsschwerpunkten der nutzenden Wissenschaftler. Digitale Veröffentlichungen - im Idealfall als E-only-Angebot sollen stärker in das überregionale System integriert werden. Zur Untermauerung der aus diesem Grunde ab dem Erwerbungsjahr 2014 umzusetzenden Profilschärfung im Bereich der Literaturerwerbung sollen als einem der ersten Schritte nicht nur in den Reihen des Beirats, sondern von allen kunstwissenschaftlichen Ordinarien in Deutschland über einen ausführlichen Fragebogen die Positionen der Fachgemeinschaft abgefragt werden. Zum einen werden dabei weitere Teilbereiche identifiziert, in denen zukünftig nur noch exemplarisch erworben werden soll oder die im Gegenteil ganz explizit als unverzichtbarer Spitzenbedarf eingestuft werden müssen. Zum anderen soll ermittelt werden, in welchen Teilbereichen der größte Bedarf an elektronisch verfügbaren Medien besteht und wo weitere Modellentwicklungen am sinnvollsten sind. Für die auf diesem Weg identifizierten Produkte sollen während der Projektlaufzeit durch das mit DFG-Förderung neu eingerichtete Kompetenzzentrum für Lizenzierung Verhandlungen mit den Verlagen geführt und die Etablierung technischer Prozesse vorangetrieben werden. Die Rolle des FID Kunst wird hierbei in kontinuierlicher Abstimmung mit der Fachcommunity die inhaltliche Steuerung sein.

Beim Gros der kleineren Zeitschriftenverlage ist davon auszugehen, dass der Erwerb und die überregionale Bereitstellung elektronischer Versionen kunstwissenschaftlicher Literatur im Sinne von Fachcommunity-Lizenzen noch auf längere Sicht nicht umsetzbar sein werden. Bei den Verhandlungen ist zudem die besondere Struktur der kunsthistorischen Fachcommunity im Auge zu behalten. Diese ist - über die Angehörigen universitärer Einrichtungen hinaus - zum einen durch eine große Zahl von Wissenschaftlern charakterisiert, die an Spezialbibliotheken unterschiedlichster Größenordnung angebunden sind (z.B. an Museen), und umfasst zum anderen eine Vielzahl von überhaupt nicht institutionell verorteten Forschern. Für zu etablierende Authentifizierungsstrukturen muss ferner berücksichtigt werden, dass zunehmend von einer interdisziplinären Nutzung kunstwissenschaftlicher Literatur auszugehen ist (z.B. arbeiten immer mehr Historiker und Medienwissenschaftler bildorientiert). 
Ergänzend zum regulären Bestandsaufbau soll künftig verstärkt der individuelle Bedarf der Wissenschaft mit Mitteln der Patron Driven Acquisition (PDA) bedient werden. So soll die schon immer bestehende Möglichkeit des „Anschaffungsvorschlags“, dessen Funktionalitäten durch die Integration in ein automatisches Workflowprogramm verbessert wird, durch eine gut sichtbare Verankerung in arthistoricum.net aktiv beworben werden. Flankiert werden soll dies durch die Konzeption und Realisierung eines PDA-Modells für gedruckt erschienene, bislang nicht erworbene Kunstliteratur, deren Metadaten über die Suche von arthistoricum.net recherchierbar gemacht werden. Das Ziel dieser Maßnahmen ist, in kürzester Zeit auf die individuellen Wünsche der Fachwissenschaftler einzugehen und zusätzlich benötigte Titel $a d$ hoc zu beschaffen.

\subsection{Im Fokus: „Open Access“}

Aufgrund der derzeit noch unbefriedigenden Marktsituation im Bereich kommerzieller E-Ressourcen sehen die UB Heidelberg und die SLUB Dresden den strategischen Schwerpunkt bei der Herstellung überregionaler OnlineVerfügbarkeit zum aktuellen Zeitpunkt im Ausbau der digitalen Bereitstellung älterer bzw. aktueller kunstwissenschaftlicher Literatur im Open Access. Neben der quantitativen Erweiterung der Publikationsplattform ART-Dok im Bereich der elektronischen Erst- und Zweitpublikation sollen neue Wege zur dynamischen Einbindung der Veröffentlichungen in externe Umgebungen (z. B. Webseiten der Autoren, Themenportale in arthistoricum.net) geschaffen werden. Ebenfalls ausgebaut werden soll die Bereitstellung der technischen Infrastruktur für die Publikation von OpenAccess-Zeitschriften beziehungsweise Monografien oder Serien. Im Fokus soll dabei dieSichtbarkeit der Herausgeber durch flexible Anpassungsmöglichkeiten an das jeweilige Corporate Design, die Gewährleistung der Zitierfähigkeit sowie die Optimierung des Nachweises in nationalen und internationalen Nachweisinstrumenten stehen. Verschiedene Kooperationsanfragen liegen bereits vor und belegen dieZukunftsträchtigkeit dieses Aktionsfelds.

Ergänzend zu den bislang an der UB Heidelberg durchgeführten, überwiegend thematisch ausgerichteten Projekten zur Digitalisierung kunstwissenschaftlicher Literatur sollen nun weitere Auswahlstrategien treten: Über die Etablierung des „Digitalen Wunschbuchs“ sollen spezifische und forschungsnahe Einzelwünsche aus der Fachcommunity direkt erfüllt werden. Neben dem Wunsch nach Digitalisierung gemeinfreier Monografien kann es sich hierbei auch um das Ziel der Onlinestellung der eigenen Schriften handeln. In enger Kooperation mit verschiedenen wissen- schaftlichen Forschungsprojekten sollen kleinere, abgeschlossene Literaturkonvolute zu spezifischen Forschungsbereichen digitalisiert und individuell präsentiert werden. Auch hier liegt ein Schwerpunkt auf aktueller Literatur, für die einfache Nutzungsrechte eingeholt werden sollen. Zudem soll eine vertiefende Erschließung bereits digitalisierter Materialien durch Georeferenzierungen oder durch reziproke semantische Vernetzung vorgenommen werden.

Ein weiterer Arbeitsschwerpunkt soll sich - unter Bezug auf Anforderungen der kooperierenden Wissenschaftler - dem Ausbau der Funktionalitäten des Heidelberger Workflowsystems widmen. Hierbei zielen alle Maßnahmen auf die Verbesserung der Möglichkeiten zur vernetzenden Tiefenerschließung in Kooperation mit der Wissenschaft. Leitgedanke ist hier die interaktive Mitarbeit externer Wissenschaftler im Sinne einer virtuellen Forschungsumgebung sowie die Verbesserung der internationalen Referenzierbarkeit.

Obwohl der gesamte Zeitraum der Kunst nach 1945 unter die Schutzfristen des Urheberrechts fällt und die Möglichkeiten der Digitalisierung und Online-Bereitstellung von Literatur und von Primärquellen somit deutlich eingeschränkt sind, hat sich die SLUB Dresden in den zurückliegenden Jahren über Vereinbarungen mit den Verwertungsgesellschaften Bild-Kunst sowie Wort erfolgreich um individuelle Lösungsmöglichkeiten bemüht. Dies gilt für den Bildbestand der Deutschen Fotothek sowie projektbezogen auch für retrospektive Digitalisierungsvorhaben. Dieses funktionierende Modell soll konsequent auf weitere Bereiche ausgedehnt werden. Thematische Schwerpunkte bildeten die Kunst der DDR und der osteuropäischen Nachbarländer, der sich Kunstgeschichte und Kunstsoziologie und insbesondere auch die Geschichte der Fotografie im letzten Jahrzehnt verstärkt zugewandt haben. Als weitere Facette der kooperativen Retrodigitalisierung sollen gemeinfreie und „verwaiste“ Werke zu den Themenbereichen Design, Reklame/Gebrauchsgrafik einschließlich Exlibris zur Unterstützung von Forschung und Lehre an Medien- und Kunsthochschulen via arthistoricum.net bereitgestellt werden.

\subsection{Von Bibliografien und Normdaten}

Zu den klassischen Aufgaben einer Sondersammelgebietsbibliothek gehörten seit Jahrzehnten die formale und sachliche Erschließung kunstwissenschaftlicher Literatur sowie die Mitarbeit an den nationalen Normdateien der Deutschen Nationalbibliothek. Diese Aktivitäten bezogen sich jedoch stets nur auf die Erschließung der erworbenen Literatur oder der bereitgestellten eigenen digitalen Medien. 
Im Kontext des Fachinformationsdienstes sollen auf konkrete Anforderungen aus der Wissenschaft hin und durchaus auch unabhängig vom eigenen Bestand Mehrwertdienste geschaffen werden, die bibliothekarische Fachkompetenz zielführend in wissenschaftliche Forschungsprojekte einbringen. So soll beispielsweise eine technische Infrastruktur für datenbankgestützte kunstwissenschaftliche Bibliografien konzipiert werden, die als vielfältig nachnutzbares Tool zur elektronischen Erfassung von Fachbibliografien eingesetzt werden kann. Angedacht ist ebenfalls die systematische Erstellung von Werkdatennormsätzen in der Gemeinsamen Normdatei (GND). Um den Einsatz diese Normvokabulars auch im Museumsbereich weiter zu etablieren und im Kontext von Linked Data nutzbar zu machen, sollen ausgehend von im Fach anerkannten Referenzwerken erstmals systematisch Werkdatensätze mit wissenschaftlich gesicherten Inhalten in der GND angelegt werden

\subsection{Im Austausch mit der Wissenschaft}

Die zukünftige Öffentlichkeitsarbeit für den Fachinformationsdienst Kunst soll auf den bereits etablierten Verbreitungs- und Kommunikationswegen aufbauen. Neben regelmäßigen Postings über die fachrelevanten Mailinglisten lassen sich neue Angebote und Dienstleistungen über den arthistoricum.net-Blog, die arthistoricum.net-Facebookseiten sowie über Twitter verbreiten. Ausbaumöglichkeiten im Hinblick auf die Einbindung von arthistoricum.net in soziale Forschernetzwerke wie beispielsweise academia. edu und researchgate.net sollen untersucht werden. Geplant ist die Durchführung eines Workshops, in dem gemeinsam mit dem Beirat, den Kooperationspartnern, Vertretern des Verbandes Deutscher Kunsthistoriker, aber auch bislang noch nicht mit dem Fachinformationsdienst zusammenarbeitenden Vertretern des Fachs sowie Nachwuchswissenschaftlern das Dienstleistungsangebot des Fachinformationsdienstes evaluiert, nachjustiert und weiterentwickelt werden soll. Erhofft werden vor allem Erkenntnisse über die zukünftigen Bedürfnisse und Anforderungen. Auch sollen hierdurch Grundlagen für weitere Kooperationen gelegt werden.

Darüber hinaus soll das neu konzipierte FID-Angebot mit seinen Dienstleistungskomponenten im Rahmen von Roadshows direkt vor Ort in Kunsthistorischen Instituten oder Forschungseinrichtungen an Wissenschaftler und Studierende vermittelt werden. Die Erfahrungen der letzten Jahre zeigten für themenspezifische Präsentationen im unmittelbaren Kontext der Anwender in der Regel positive Resonanz und erwiesen sich zugleich als geeignete Platt- formen zur Kommunikation weiterer fachspezifischer Angebote.

Die aktive Mitarbeit der FID-Verantwortlichen im Vorstand und verschiedenen Fachgruppen der Arbeitsgemeinschaft der Kunst- und Museumsbibliotheken (AKMB) sichert den Kontakt zu fast allen Kunstbibliothekaren in Deutschland, Österreich und der Schweiz, welche als ideale Multiplikatoren die Angebote des FID in die Fachcommunities an Universitäten, Fachhochschulen und Museen weiter tragen. Gleiches gilt für die vielfältigen Kontakte im Kontext der zahlreichen Kooperationsprojekte und Teilnahmen an Arbeitsgruppen.

\section{Zusammenfassung}

Mit den beschriebenen, in den letzten beiden Jahren gemeinsam entwickelten Strukturen und ihrem Ausbaupotenzial sehen sich die beiden Sondersammelgebietsbibliotheken in Dresden und Heidelberg gut gewappnet für den anstehenden Paradigmenwechsel. Nun gilt es - natürlich nur im Fall einer positiven Begutachtung des Vorhabens $^{24}$ - vor allem die Herausforderungen der digitalen Transformation anzunehmen und in einem intensiven Austauschprozess mit der kunstwissenschaftlichen Community neue Lösungswege zur Bereitstellung digitaler Veröffentlichungen und Informationsangebote zu entwickeln. Die in den neuen Förderrichtlinien vorgesehene Flexibilität beim Einsatz der Mittel bietet die Chance, unterstützt durch von der DFG gefördertes Personal die vorgesehenen Aktionsfelder zur Verbesserung der Informationsversorgung für die kunstgeschichtliche Forschung zu bearbeiten.

\section{Dr. Maria Effinger}

Universitätsbibliothek Heidelberg

Plöck 107-109

D-69117 Heidelberg

Effinger@ub.uni-heidelberg.de

\section{Dr. Katja Leiskau}

Sächsische Landesbibliothek -

Staats- und Universitätsbibliothek Dresden

D-01054 Dresden

Katja.Leiskau@slub-dresden.de

Annika-Valeska Walzel M.A., M.A. (LIS)

Sächsische Landesbibliothek -

Staats- und Universitätsbibliothek Dresden

D-01054 Dresden

Annika-Valeska.Walzel@slub-dresden.de

24 Der bei der DFG eingereichte Antrag wurde im Dezember 2013 uneingeschränkt bewilligt. Projektbeginn war der 1. Januar 2014. 